\title{
A la búsqueda de tendencias históricas en el consumo de vertebrados en Chiapas (México). Un estudio de caso combinando evidencias
}

\author{
Eduardo Corona-M. y Patricia EnRíquez VÁzquez \\ Centro INAH Morelos, Instituto Nacional de Antropología e Historia (México) \\ ecoroma09@gmail.com
}

Recibido: 1 de marzo de 2010

Aceptado: 1 de julio 2010

\begin{abstract}
RESUMEN
Desde la época prehispánica y hasta la actualidad en el Estado de Chiapas se registra el consumo de diferentes especies animales, una práctica aún frecuente entre los grupos indígenas y rurales. Nuestra intención es obtener una primera aproximación para identificar qué animales se han preferido para el consumo. En este trabajo se analizan de manera comparativa evidencias arqueozoológicas y resultados de un trabajo de campo etnozoológico en las zonas Altos y Selva del Estado de Chiapas; con base en ello se obtiene un listado global de 64 taxones de vertebrados con indicios de haber sido consumidos, varios de ellos identificados tanto en las localidades antiguas como en las recientes. De esta manera se obtiene una perspectiva histórica que nos permite medir el cambio y la persistencia en el aprovechamiento de los recursos faunísticos.
\end{abstract}

Palabras clave: Chiapas, arqueozoología, etnozoología, relaciones hombre-fauna, consumo.

\section{In Search of Historical Trends on Vertebrate Consumption in Chiapas (Mexico). A Study Case Combining Evidences}

\section{ABSTRACT}

From the Pre-Hispanic epoch onward were recorded the use of many different animal species, and currently is a common practice by rural and indigenous groups. Our aim is obtain a first approximation to identify the animals preferred for consumption. In this paper were analyzed, in a comparative way, the archaeozoological and ethnozoological data from Altos and Selva regions located at the State of Chiapas. A global list of 64 vertebrates with consumption evidences was obtained. Some of them were found both in ancient and current localities. By this way was obtained an historical perspective to measure the change and persistence in the use of faunal resources.

Keywords: Chiapas, archaeozoology, ethnozoology, human-animal relationship, consumption.

Sumario: 1. Introducción. 2. Métodos. 3. Resultados y discusión. 4. Conclusiones. 5. Referencias bibliográficas.

\section{Introducción}

Las interacciones de los seres humanos con los animales se manifiestan en una serie de patrones utilitarios, los cuales pueden adquirir rasgos culturales característicos que pueden ser variables en el tiempo, ya que se encuentran vinculados a la disponibilidad de la fauna y a la actitud cultural. Algunos son básicos para la supervivencia, como los de uso alimentario o los utilizados como materia prima para la elaboración de herramientas. Mientras que otros corresponden a la esfera de la elaboración ideo- 
lógica, donde los recursos faunísticos se incorporan a las explicaciones que sobre el mundo han elaborado las diversas sociedades, mediante la asignación de atributos o valores religiosos o rituales. El análisis de estas relaciones entre el hombre y la fauna constituye un campo de múltiples contactos entre la Biología, la Arqueología y la Antropología (Corona-M. y Arroyo Cabrales 2003, 2007).

Desde la época prehispánica ha sido frecuente el consumo de diferentes especies animales; en la actualidad este hecho es aún frecuente entre los grupos indígenas y rurales debido, principalmente, a la situación económica que enfrentan. Entre los grupos que habitaron el área maya, se han hecho diversos intentos por identificar las arqueofaunas y reconstruir sus dietas (véase Emery 2004a y las referencias ahí señaladas); en general, se coincide en que los mayas antiguos no consumían demasiada carne, ya que sólo lo hacían en fiestas y reuniones donde se le brindaba un carácter religioso y mítico (Emery 2004a, 2004b; Marmolejo 2000). Se considera, además, que en la cultura maya las actividades adicionales más importantes para el sustento eran la caza y la pesca, por lo que fue necesario desarrollar y perfeccionar éstas prácticas con el fin de que fueran exitosas y se elaboraron instrumentos como flechas, cerbatanas y trampas para capturar animales terrestres; para la pesca se utilizaban arpones con punta de obsidiana en forma de aguijón dentado, o una especie de anzuelo que se fabricaba de huesos o espinas (Marion 1994; Sánchez Álvarez 2000; Soto Hall 1941). Actualmente, entre los grupos mayenses que habitan Chiapas el consumo de carne es más cotidiano que en la época prehispánica (Guerra 2001; Marmolejo 2000).

En Chiapas se han efectuado algunos estudios que reconocen la importancia de los recursos animales en la subsistencia humana desde el Precerámico al Posclásico (Agrinier 1975; Chávez 1969; Flannery 1969; García Bárcena 1982; Green y Lowe 1967; Marrinan 1986; Martínez Muriel 1989; Voorhies 1976). Sin embargo, los estudios comparativos sobre los cambios y las persistencias en el aprovechamiento cultural de especies animales son un tema ausente en la agenda de etnobiólogos y arqueozoológos; no obstante, se comienza a llamar la atención sobre este aspecto y se observa la necesidad de abordarlo en forma transdisciplinar (Harris 2006).

En este trabajo nos planteamos efectuar una aproximación al estudio sobre el cambio y la persistencia en el uso de los recursos animales, basado en las evidencias que provienen tanto de la arqueozoología como de la etnozoología para dos zonas de Chiapas: Altos y Selva.

\section{Métodos}

Aun cuando en Chiapas existen diversas localidades arqueozoológicas, se seleccionaron sólo siete de ellas (Figura 1). Dos son las más antiguas: Santa Marta (Álvarez 1976) y Aguacatenango (García Bárcena 1982); ambas se encuentran cercanas a las zona de estudio y sirven de referencia por corresponder al período Precerámico (PC), por lo que se ha creado un grupo particular para ellas. Las cinco restantes son San Cristóbal de las Casas (Álvarez 1979, 1980), que es la única que se ubica en la región de los Altos, mientras que en la región Selva se encuentran Palenque (Ocaña 1997), Yaxchilán (Soto Toral 1998), Toniná (Álvarez 1982; Álvarez et al. 1980, 1990; Ocaña 
y Polaco 1982, 1990) y Laguna Miramar (Polaco 1989). Estas cinco últimas abarcan temporalidades del Clásico al Postclásico, es decir son prehispánicas y se ubicaron en un grupo genérico al que denominamos arqueozoológico (AZ). Para la etapa Colonial no se obtuvieron registros del consumo de fauna, ya fuese en las crónicas históricas o en las Relaciones Geográficas que comprendían el área de estudio. Por su parte la información etnozoológica proviene de 68 entrevistas de campo efectuadas en poblados ubicados en la zona de los Altos y Selva (Enríquez 2005; Enríquez et al. 2006).

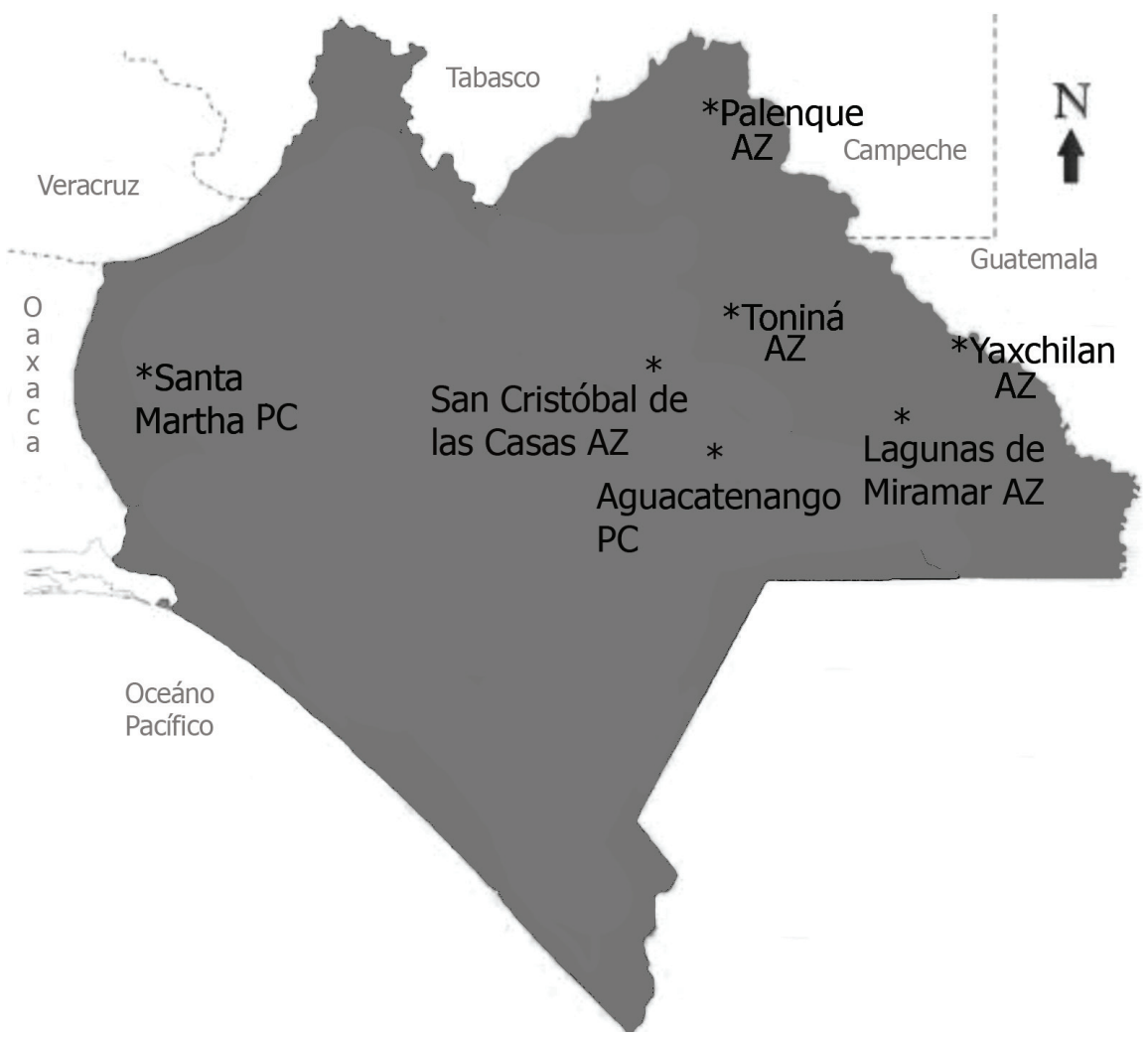

Figura 1: Mapa del Estado de Chiapas (México) con la ubicación aproximada de las localidades mencionadas en el texto. (Las siglas representan la cronología: $\mathrm{PC}=$ precerámico, $\mathrm{AZ}=$ arqueozoológico).

El trabajo se concentra en los vertebrados terrestres y, para fines taxónomicos, se basa en los trabajos de Flores-Villela (1993) para la herpetofauna, Howell y Webb (1995) para aves y Reid (1997) para mamíferos.

La comparación entre fuentes se basa en las listas de especies que en la información etnozoológica (EZ) de la región Altos y Selva se identificaban como de fines alimentarios y con los listados de fauna de las localidades de los períodos precerámico $(\mathrm{PC})$ y arqueozoológico (AZ), ya indicadas. Sin embargo, debe destacarse que en algunos casos el uso de estos animales no es únicamente alimentario, ya 
que también puede adquirir características rituales, medicinales o como materia prima en la elaboración de artefactos, como se ha observado en otras áreas culturales (Corona-M. 2005; Corona-M. y Arroyo Cabrales 2003). Sin embargo, no es nuestra intención en este trabajo determinar si los animales, sobre todo en aquellos que son restos arqueofaunísticos, fueron procesados para un fin específico no alimentario, ya que nuestro punto focal son los vertebrados usados como recurso natural.

Por otro lado, se debe destacar que este tipo de trabajos comparativos se basan en la búsqueda de denominadores comunes dentro de una diversidad de enfoques, de resultados presentados, de las formas de obtener los datos e, incluso, de filosofías de trabajo. En este caso, las listas de fauna identificada fueron la base adoptada para obtener una primera aproximación diacrónica sobre el consumo de los vertebrados. Sin embargo, se reconocen diversas limitaciones en este enfoque, puesto que el registro arqueozoológico se encuentra afectado por procesos que van desde los aspectos naturales relativos a la distribución geográfica de las especies, la preservación de los restos, las técnicas de recuperación arqueológica o las posibilidades de identificación, tanto osteológica como en el campo, de esas especies, aspectos que se hacen más complejos en algunos de los grupos de microfauna de vertebrados, como son los anfibios, reptiles y aves.

En el caso particular de nuestras identificaciones, cuando se observó que había más de una especie en el área de estudio o que los datos proporcionados no permitían una identificación adecuada, se procedió a adoptar un criterio conservador, asignándolo al taxón inmediatamente superior, sea género o familia.

Para atenuar varios de los efectos antes descritos se adoptó un método comparativo inter-localidades basado en el índice de diversidad taxonómica (IDT) establecido por Clarke y Warwick (1998) e implementado por el programa computacional PAST (Hammer 2009). Las ventajas del índice referido es que permite la comparación de localidades mediante listados taxonómicos; no depende del tamaño de la muestra, excepto en aquellos casos donde esta es muy pequeña -menos de cinco taxones-, que no fue nuestro caso. Además, proporciona los valores del índice con límites de confianza del 95\%. De este modo, los valores que se encuentran dentro de dichos límites nos sugieren que las localidades son aptas para la comparación.

Los datos taxonómicos se procesaron también como matrices binarias para la aplicación de dos métodos multivariantes: la seriación y el dendrograma, los cuales también forman parte del programa computacional PAST (Hammer 2009). El primero nos permite encontrar tanto los taxones exclusivos como los comunes, respecto a las temporalidades y las zonas analizadas. El segundo permite medir la similitud entre las localidades a partir de su contenido taxonómico; para ello se usó el índice de Raup y Crick como medida de distancia, pues permite evaluar los datos observados contra una distribución esperada construida con todos los registros de taxones a los que se les aplica una serie de réplicas aleatorias (bootstrap) y así formar los grupos con el algoritmo UPGMA (Unweighted Pair-Group Moving Average), que constituye uno de los procedimientos más comunes. Los resultados obtenidos se miden de cero (sin similitud) a uno (similitud total). 
En estas comparativas se consideraron las localidades ya señaladas previamente y dos zonas etnozoológicas: Altos y Selva.

\section{Resultados y discusión}

En total se encontraron 64 taxones de vertebrados, de los cuales son: cuatro de anfibios, 15 de reptiles, 10 de aves y 35 de mamíferos, ubicados en los distintos períodos cronológicos (Cuadro 1).

Cuadro 1: Listado taxonómico de los vertebrados terrestres incluidos en el análisis.

\begin{tabular}{|c|c|c|c|c|c|c|}
\hline CNS & Clase & Orden & Familia & Taxón identificado & Nombre común & Período* \\
\hline 1 &.$\pi$ & Anura: & Bufonidae: & Bufo sp. & Sapo & AZ, EZ \\
\hline 2 & 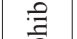 & & Hylidae: & Smilisca baudini & Rana arborícola & EZ \\
\hline 3 & 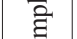 & & Ranidae: & Rana sp. & Rana & $\mathrm{AZ}, \mathrm{EZ}$ \\
\hline 4 & 之 & & Rhinophrynidae: & Rhinophrynus dorsalis & Sapo excavador & EZ \\
\hline 5 & & Crocodylia: & Crocodylidae: & Crocodylus moreletii & Cocodrilo de pantano & $\mathrm{AZ}, \mathrm{EZ}$ \\
\hline 6 & & Squamata: & Anguidae: & Mesaspis moreleti & Lagarto escorpión & EZ \\
\hline 7 & & & Corytophanidae: & Basiliscus vittatus & Basalisco & EZ \\
\hline 8 & & & & Corytophanes sp. & Turipache & EZ \\
\hline 9 & & & Iguanidae: & Ctenosaura sp. & Garrobo & PC, EZ \\
\hline 10 & & & & Iguana iguana & Iguana verde & PC, EZ \\
\hline 11 & $\stackrel{\mathbb{Z}}{=}$ & & Phrynosomatidae & Sceloporus sp. & Lagartija escamosa & EZ \\
\hline 12 & 蒙 & & Polychrotidae: & Anolis sp. & Lagartija o abaniquitos & $\mathrm{AZ}, \mathrm{EZ}$ \\
\hline 13 & $\approx$ & & Teiidae: & Ameiva undulata & Lagartija metálica & $\mathrm{EZ}$ \\
\hline 14 & & & Boidae: & Boa constrictor & Boa & EZ \\
\hline 15 & & & Viperidae: & Crotalus durissus & Víbora de cascabel & EZ \\
\hline 16 & & Testudines: & Chelydridae: & Chelydra serpentina & Tortuga cocodrilo & EZ \\
\hline 17 & & & Dermatemydidae: & Dermatemys mawii & Tortuga riverina & $\mathrm{AZ}, \mathrm{EZ}$ \\
\hline 18 & & & Emydidae: & Trachemys scripta & Jicotea & PC, AZ, EZ \\
\hline 19 & & & Kinosternidae: & Kinosternon sp. & Tortuga casquito & $\mathrm{PC}, \mathrm{AZ}, \mathrm{EZ}$ \\
\hline 20 & & Falconiformes: & Accipitridae: & Buteo sp. & Águila & $\mathrm{AZ}$ \\
\hline 21 & & & Falconidae: & Falco sp. & Halcon & $A Z$ \\
\hline 22 & & Galliformes: & Cracidae: & Ortalis sp. & Chachalaca & AZ, EZ \\
\hline 23 & & & & Penelope purpurascens & Pava cojolita & $\mathrm{PC}, \mathrm{EZ}$ \\
\hline 24 & \& & & & Crax rubra & Hocofaisán & $\mathrm{PC}, \mathrm{AZ}, \mathrm{EZ}$ \\
\hline 25 & 永 & & Phasianidae: & Meleagris sp. & Guajolote & $\mathrm{AZ}, \mathrm{EZ}$ \\
\hline 26 & & & Odontophoridae: & & Codorniz & $\mathrm{AZ}, \mathrm{EZ}$ \\
\hline 27 & & Columbiformes: & Columbidae: & & Paloma & $\mathrm{EZ}$ \\
\hline 28 & & Apodiformes: & Trochiilidae: & & Colibrí & EZ \\
\hline 29 & & Passeriformes: & Icteridae: & Quiscalus mexicanus & Zanate & EZ \\
\hline 30 & & Didelphimorphia: & Didelphidae: & Didelphis sp. & Tlacuache & $\mathrm{AZ}, \mathrm{EZ}$ \\
\hline 31 & & Xenarthra: & Dasypodidae: & Dasypus novemcinctus & Armadillo & $\mathrm{PC}, \mathrm{AZ}, \mathrm{EZ}$ \\
\hline 32 & $\stackrel{\pi}{=}$ & Primates: & Cebidae: & Allouatta sp. & Saraguato & $\mathrm{AZ}, \mathrm{EZ}$ \\
\hline 33 & $\bar{\Xi}$ & & & Ateles geoffroyi & Mono araña & AZ, EZ \\
\hline 34 & Е & Rodentia: & Sciuridae: & Sciurus sp. & Ardilla & $\mathrm{PC}, \mathrm{AZ}, \mathrm{EZ}$ \\
\hline 35 & $\sum^{\pi}$ & & Geomyidae: & Orthogeomys sp. & Tuza & $\mathrm{AZ}, \mathrm{EZ}$ \\
\hline 36 & & & Heteromyidae: & Liomys sp. & Ratón espinoso & $\mathrm{PC}$ \\
\hline 37 & & & & Heteromys sp. & Ratón espinoso de bosque & AZ, EZ \\
\hline 38 & & & Muridae: & Oryzomys sp & Rata arrocera & $\mathrm{PC}, \mathrm{AZ}$ \\
\hline 39 & & & & Sigmodon hispidus & Rata algodonera & $\mathrm{PC}, \mathrm{AZ}$ \\
\hline 40 & & & & Tylomys sp. & Ratón tlacuache & $\mathrm{AZ}, \mathrm{EZ}$ \\
\hline
\end{tabular}




\begin{tabular}{|c|c|c|c|c|c|c|}
\hline 41 & & & & Ototylomys sp. & Rata orejona & PC, AZ, EZ \\
\hline 42 & & & & Neotoma mexicana & Rata de bosque & $\mathrm{AZ}, \mathrm{EZ}$ \\
\hline 43 & & & & Baiomys sp. & Ratón pigmeo & $\mathrm{PC}$ \\
\hline 44 & & & & Reithrodontomys sp. & Ratón cosechero & $\mathrm{PC}, \mathrm{AZ}, \mathrm{EZ}$ \\
\hline 45 & & & & Peromyscus sp. & Ratón canguro & $\mathrm{PC}, \mathrm{AZ}, \mathrm{EZ}$ \\
\hline 46 & : & & Erenthizontidae: & Coendou mexicanus & Puerco espin & EZ \\
\hline 47 & $\Xi$ & & Dasyproctidae: & Dasyprocta sp. & Guaqueque & AZ, EZ \\
\hline 48 & $\stackrel{\Xi}{\pi}$ & & Agoutidae: & Aguti paca & Tepezcuintle & $\mathrm{PC}, \mathrm{AZ}, \mathrm{EZ}$ \\
\hline 49 & & Lagomorpha: & Leporidae: & Sylvilagus sp. & Conejo & PC, AZ, EZ \\
\hline 50 & & Carnivora: & Canidae: & Canis familiaris & Perro & $\mathrm{AZ}, \mathrm{EZ}$ \\
\hline 51 & & & Procyonidae: & Procyon lotor & Mapache & EZ \\
\hline 52 & & & & Nasua narica & Tejón & PC, EZ \\
\hline 53 & & & Mustelidae: & Mustela frenata & Comadreja & EZ \\
\hline 54 & & & & Mephitis macroura & Zorrillo rayado & $\mathrm{AZ}, \mathrm{EZ}$ \\
\hline 55 & & & & Spilogale putorius & Zorrillo manchado & EZ \\
\hline 56 & & & & Conepatus sp. & Zorrillo de espalda blanca & AZ, EZ \\
\hline 57 & & & & Lutra longicaudis & Nutria & $\mathrm{AZ}, \mathrm{EZ}$ \\
\hline 58 & & & Felidae: & Puma concolor & Puma & $\mathrm{AZ}$ \\
\hline 59 & & & & Panthera onca & Jaguar & AZ, EZ \\
\hline 60 & & Perissodactyla: & Tapiridae: & Tapirus bairdii & Tapir & AZ, EZ \\
\hline 61 & & Artiodactyla: & Tayassuidae: & Tayassu tajacu & Pecari de collar & $\mathrm{PC}, \mathrm{AZ}, \mathrm{EZ}$ \\
\hline 62 & & & & Dicotyles pecari & Pecari de labios blancos & $\mathrm{AZ}, \mathrm{EZ}$ \\
\hline 63 & & & Cervidae: & Odocoileus virginianus & Venado cola blanca & $\mathrm{PC}, \mathrm{AZ}, \mathrm{EZ}$ \\
\hline 64 & & & & Mazama americana & Temazate & PC, AZ, EZ \\
\hline
\end{tabular}

* Período cronológico: PC: pre-cerámico; AZ: registro arqueozoológico; EZ registro etnozoológico.

Al contrastar las localidades en términos del índice de diversidad taxonómica, se observa que se tiene una buena base comparativa, en tanto la mayoría de las localidades están dentro de los intervalos de confianza del $95 \%$, con la excepción de dos localidades arqueozoológicas que son Palenque y San Cristóbal de las Casas (Figura 2). La razón de ello es que en estas dos muestras predominan los mamíferos, indicando alguno de los sesgos en el análisis o en la identificación que habíamos previsto en este tipo de muestras. Ante ello las opciones son las de reducir la base comparativa a sólo los mamíferos o efectuar las comparaciones pertinentes, siendo cautos en las interpretaciones al tomar en cuenta esta limitación. Nosotros tomamos la segunda opción, para evitar una pérdida de información.

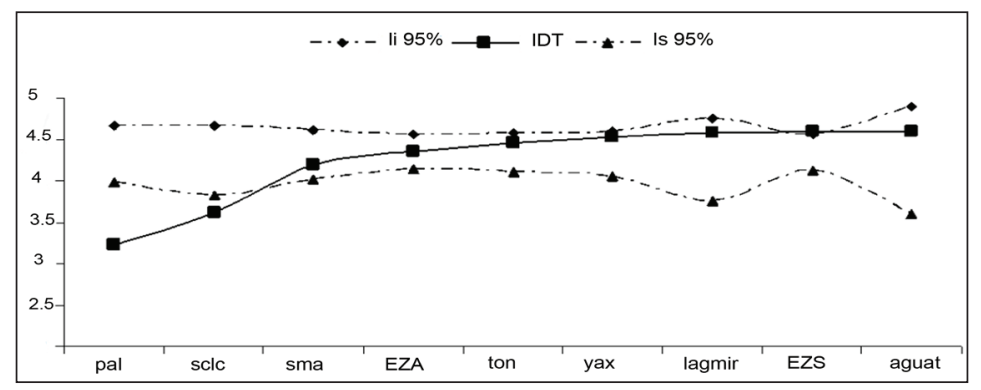

Figura 2: Gráfico comparativo de diversidad inter-localidades. IDT = Índice de diversidad taxonómica; Li 95\% = Límite inferior de confianza al 95\%; Ls 95\% = Límite superior de confianza al 95\%. PAL = Palenque, $\mathrm{SCLC}=$ San Cristóbal de Las Casas, $\mathrm{SMA}=$ Santa Marta, $\mathrm{EZA}=$ localidades etnozoológicas Altos, $\mathrm{TON}=$

Tonina, LM = Lagunas de Miramar, EZS = localidades etnozoológicas Selva, AGU = Agucatenango. 
El dendrograma nos muestra que hay un agrupamiento más incluyente en donde se encuentran las localidades del área Selva, tanto las arqueozoológicas como la etnozoológica, además de las precerámicas, todo ello con una similitud mayor del $60 \%$; es decir, nos está sugiriendo que hay varios elementos faunísticos que les son comunes a lo largo del tiempo. Otro grupo es el que se forma con las localidades de los Altos; lo atípico es que aquí se agrupa la localidad de Palenque, que en realidad es del área Selva, lo que manifiesta uno de los sesgos ya mencionados, al ser esta una de las localidades donde sólo se registran mamíferos.

También debe destacarse que la similitud de las localidades dentro de cada uno de los grupos ronda el $90 \%$. Es decir, que se refuerza la idea de que existe una fuerte base común en los animales registrados, y por ende utilizados, además de que varios de estos elementos persisten en el tiempo, pero también nos sugiere que pueden existir ciertas tendencias o particularidades regionales, las cuales intentaremos esclarecer a continuación.

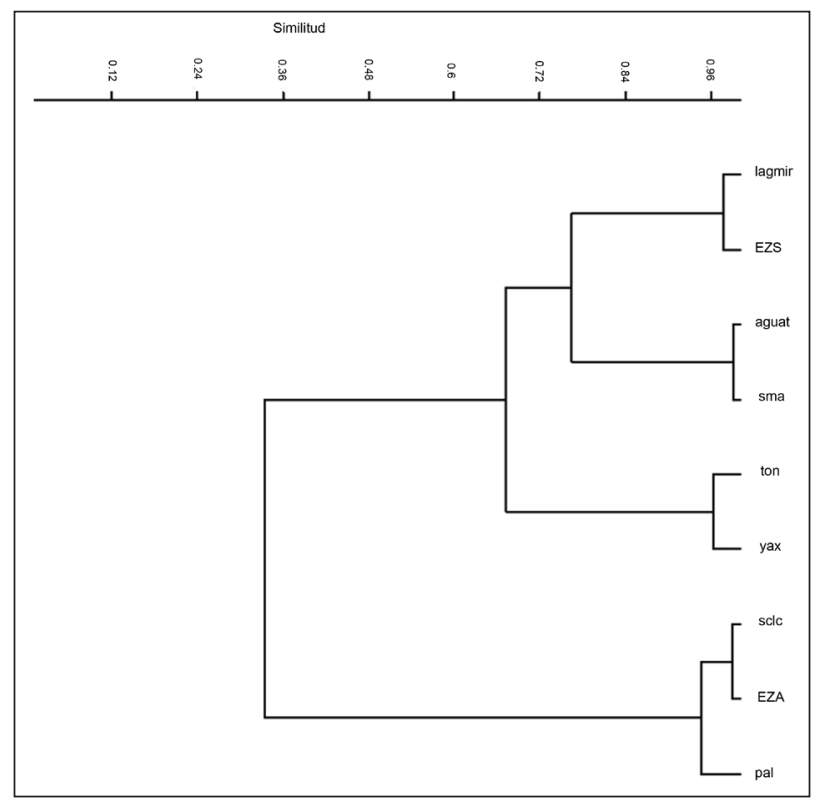

Figura 3: Dendrograma basado en el índice de Raup-Crick y el algoritmo UPMGA, muestra la similitud de las localidades con base en su contenido taxonómico. PAL $=$ Palenque, $\mathrm{SCLC}=\mathrm{San}$ Cristóbal de Las Casas, SMA = Santa Marta, EZA = localidades etnozologicas Altos, TON = Tonina, LM = Lagunas de Miramar, EZS = localidades etnozologicas Selva, AGU = Agucatenango.

$\mathrm{Al}$ analizar la proporción de vertebrados con base en los grupos cronológicos y regionales (Figura 4) destaca nuevamente el caso del registro arqueozoológico de la región Altos, que se encuentra sesgado hacia los mamíferos. Sin embargo, también se observa una importante similitud entre el grupo precerámico y el etnozoológico de los Altos, ya que, con la excepción de los anfibios, el resto de los vertebrados tiene proporciones similares. Esta ausencia de los anfibios en la época más antigua puede 
sugerir tanto un sesgo en la recuperación o bien que no eran un recurso utilizado, aspecto que no podemos discernir con la evidencia disponible. Pero también nos sugiere que, en términos de las proporciones indicadas, hay señales de la persistencia en el consumo de esos grupos de vertebrados en todos los períodos.

Por su parte en la región Selva, para las dos cronologías que se registran, se observa un uso diversificado de la fauna, donde se encuentran representados los cuatro grupos de vertebrados, aunque en el periodo actual se ve una ligera disminución en el uso de los mamíferos. Ello puede indicar cambios en su disponibilidad, o bien cambios en la actitud cultural hacia ellos, es decir son menos apreciados debido a un uso más intensivo de los actuales animales domésticos, lo que reduce la diversidad de fauna utilizada.

Las diferencias entre las proporciones de vertebrados en las regiones Selva y Altos, donde la primera tiene un patrón más diversificado, sugiere una influencia de las circunstancias ambientales, ya que es claro que en la primera región existe una mayor cantidad de especies de las cuales disponer.

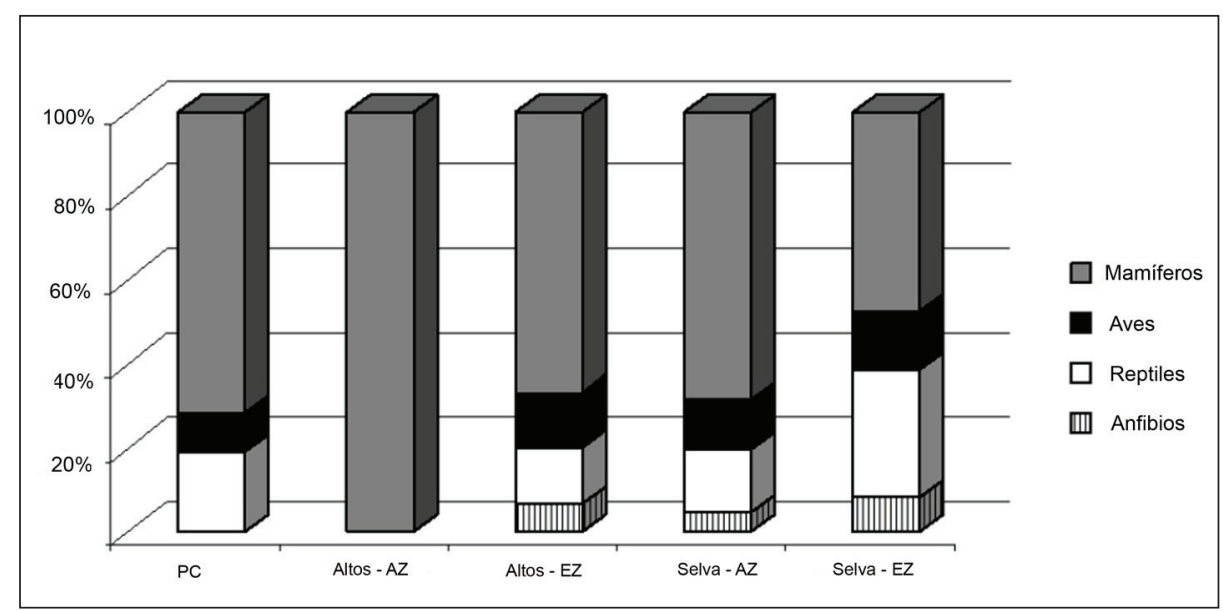

Figura 4: Gráfico que muestra la proporción de vertebrados terrestres usados por temporalidad y por región. Clave de temporalidad, $\mathrm{PC}=$ precerámico, $\mathrm{AZ}=$ arqueozoológico, $\mathrm{EZA}=$ etnozoológico Altos, EZS $=$ etnozoológico Selva.

Dados los elementos anteriores -que nos indican la existencia de una base común de animales usados a lo largo del tiempo, pero también de cambios en dichos usos-, se utilizó el análisis de seriación para definir aquellos organismos que pudieran ser diagnósticos de cada período, de tal suerte que los animales exclusivos son aquellos que sólo aparecen en un período cronológico determinado y nos sugieren que hay cambios en el uso de la fauna, ya que pudieron ser un recurso temporal o bien representar un cambio en la actitud cultural hacia esos organismos. Esta exclusividad puede ser interpretada de dos maneras: si sólo se encuentran en el registro arqueozoológico, es una pérdida para el aprovechamiento, y si se encuentran en listas etnozoológicas, se puede tomar como una incorporación al uso. La persistencia se evalúa con aquellos 
organismos que aparecen como una constante en todos los períodos cronológicos aquí propuestos, por lo que estos recursos faunísticos pueden interpretarse como la base alimentaria histórica y común de los grupos sociales en el área de estudio.

Cabe precisar que las causas de estos cambios y persistencias en el aprovechamiento de la fauna no son, por lo pronto, materia de esta discusión, pero consideramos que este tipo de análisis son puntos de partida necesarios para ulteriores consideraciones.

Cuadro 2: Resumen de animales exclusivos por temporalidad y región y de animales con uso persistente.

\begin{tabular}{|c|c|c|c|}
\hline \multicolumn{2}{|c|}{ Precerámico } & \multicolumn{2}{|c|}{ Selva Arqueozoológico } \\
\hline Taxón & Nombre común & Taxón & Nombre común \\
\hline Lyomis sp. & ratón espinoso & Buteo sp. (Accipitridae) & águilas \\
\hline \multirow[t]{2}{*}{ Baiomys sp. } & ratón pigmeo & Falco sp. (Falconidae) & halcones \\
\hline & & Puma concolor & puma \\
\hline \multicolumn{2}{|c|}{ Altos Etnozoológico } & \multicolumn{2}{|c|}{ Selva Etnozoológico } \\
\hline Taxón & Nombre común & Taxón & Nombre común \\
\hline Mesaspis moreleti & lagartija & Smilisca baudini & rana arborícola \\
\hline Sceloporus sp. & lagartija escamosa & Rhinophrynus dorsalis & sapo excavador \\
\hline Crotalus durissus & víbora de cascabel & Basiliscus vittatus & basilisco \\
\hline Columbidae & palomas & Corytophanes sp. & turipache \\
\hline Trochiilidae & colibrí & Ameiva undulata & lagartija metálica \\
\hline Quiscalus mexicanus & zanate & Boa constrictor & boa \\
\hline Mustela frenata & comadreja & Chelydra serpentina & tortuga cocodrilo \\
\hline Spilogale putorius & zorrillo manchado & Coendou mexicanus & puerco espín \\
\hline Procyon lotor & mapache * & Procyon lotor & mapache $*$ \\
\hline \multicolumn{4}{|c|}{ Uso persistente } \\
\hline Taxón & Nombre común & Taxón & Nombre común \\
\hline Trachemys scripta & jicotea & Peromyscus sp. & ratón canguro \\
\hline Kinosternon sp. & tortuga casquito & Aguti paca & tepezcuintle* \\
\hline Crax rubra & hocofaisán & Sylvilagus sp. & conejo * \\
\hline Dasypus novemcinctus & armadillo* & Tayassu tajacu & pecari collar \\
\hline Sciurus sp. & ardilla * & Odocoileus virginianus & venado cola blanca * \\
\hline Ototylomys sp. & rata orejona & Mazama americana & temazate \\
\hline Reithrodontomys sp. & ratón cosechero & & \\
\hline
\end{tabular}

Como se observa en el Cuadro 2 (parte superior), las pérdidas en el aprovechamiento se concentran en las localidades del Precerámico y del registro arqueozoológico de la Selva, mientras que en la región Altos no se encontró ningún registro de pérdida. Esto último debe ser objeto de atención en futuras investigaciones sobre los aprovechamientos de fauna en esta área, ya que puede ser efecto de los sesgos ya referidos o bien de una persistencia en el aprovechamiento de los vertebrados en esa zona.

Cabe destacar que en esta interpretación del uso de la fauna, el hecho de que algún organismo desaparezca del registro histórico, es decir de los contextos culturales, no necesariamente debe considerarse como una reducción de la diversidad biológica en las áreas estudiadas. 
De manera particular, se registra la pérdida en el uso de dos especies de ratones (Lyomis sp. y Bayomis sp.), para el período Precerámico (Cuadro 2, superior izquierda). Este caso es interesante, ya que los roedores comúnmente se consideran intrusivos de los contextos arqueozoológicos; sin embargo, por la información etnozoológica obtenida en las entrevistas y en otras fuentes (Barragán 2001), se observa que este es un recurso alimentario común, aunque aprovechando otras especies. Por tanto, es probable que el registro de estos ratones en las localidades del Precerámico indique una disponibilidad local, y también que no eran un recurso prioritario.

Las pérdidas de registro en la región Selva (Cuadro 2, superior derecha) se concentran en las aves rapaces, como son las águilas (Buteo sp.) y los halcones (Falco sp.), aunque en ambos casos tampoco se pueden descartar la inclusión de otras especies de las familias accipitridae y falconidae. De los mamíferos se encuentra el puma (Puma concolor). El carácter simbólico de las aves rapaces y de este felino está bien documentado en la región maya. Sin embargo, es menos conocido que estas aves, en el centro de México, eran consumidas por sus supuestas propiedades medicinales (Corona-M. 2005), mientras que el puma era usado como materia prima para la elaboración de diversos objetos suntuarios (Corona-M. 2008). Tal vez fuesen adoptados dichos usos en estas regiones de Chiapas y el cambio en la actitud cultural hacia ellos, tal vez inducido por el proceso de Conquista en la región al asociarlos a prácticas paganas (Acosta 1962), les fue restando importancia cultural y, por ende, se reduciría o se abandonaría su aprovechamiento.

En el futuro, ambas situaciones deberán contrastarse con más y con otras evidencias de su posible aprovechamiento, como pueden ser las marcas de corte, de cocción $\mathrm{u}$ otras que se consideren pertinentes.

Las incorporaciones o registros exclusivos en ambas localidades etnozoológicas, si bien son de los cuatro grupos de vertebrados terrestres (Cuadro 2, parte media), predominan las de herpetofauna, mientras que en menor proporción se encuentran las de aves (palomas, colibríes y el zanate) y mamíferos (puerco espín, comadreja y zorrillo manchado). Un caso particular es el del mapache (Procyon lotor), ya que sólo se encuentra en el registro etnozoológico, pero en ambas regiones, lo que nos sugiere su amplia disponibilidad en las zonas de estudio. Es interesante anotar que en las crónicas escritas en la época colonial para el centro de México, la mayoría de estos vertebrados también tienen registros de consumo, sea alimentarios o medicinales, pero con especies distintas (Corona-M. 2005; Enriquez et al. 2006). Esto nos podría sugerir la idea de que se prefiera el consumo de organismos locales similares, lo que puede verse como una adaptación local de ese tipo de aprovechamientos, o bien que sean diversificaciones recientes de las estrategias de uso de la fauna circundante por parte de las poblaciones locales, lo que se expresa en un incremento de las especies que se cazan. Sin embargo, una investigación de mayor aliento nos podrá aportar otros elementos para interpretar más claramente estos datos.

En el caso de la persistencia, encontramos 13 vertebrados (ver Cuadro 2, sección inferior) que comprenden dos especies de tortugas, un ave gallinácea, tres roedores, tres ungulados, una ardilla y un conejo. De ellos también se puede decir que unos son característicos de la región Altos, como son los ratones -cosechero y canguro-, el conejo, la ardilla y el venado cola blanca; mientras que en la Selva predominan las 
tortugas -jicotea y casquito-, el ave, el temazate, el pecarí labios blancos y el armadi1lo. Estos son los animales silvestres que se pueden considerar la base alimentaria de estas zonas, siendo más diversa, por razones naturales, en la región Selva.

De dichas especies, cinco son las de mayor presencia en los tres períodos cronológicos establecidos, por lo que se convierten en denominadores comunes de consumo; estas son el armadillo (Dasypus novemcinctus), la ardilla (Sciurus sp.), el tepezcuintle (Aguti paca), el conejo (Sylvilagus sp.) y el venado cola blanca (Odocoileus virginianus). Todos ellos son mamíferos de talla mediana a grande que, debido a su aporte cárnico y las abundantes poblaciones, son fáciles objetivos de caza en las regiones de estudio. Estas cinco especies pueden interpretarse como la existencia de un núcleo básico de fauna consumida, mientras que el resto de las especies constituyen variantes y objetivos ocasionales, que pueden estar en función de la disponibilidad en los alrededores de los núcleos poblacionales.

\section{Conclusiones}

Con los datos disponibles, que cubren de manera general tres grandes períodos cronológicos y mediante un análisis comparativo que confronta varios de los problemas que derivan de los análisis que se han efectuado previamente, se obtiene una aproximación inicial sobre el aprovechamiento histórico de los recursos faunísticos en dos regiones del Estado de Chiapas.

Se han asumido una serie de métodos que nos pueden ayudar a mitigar los efectos de varios problemas propios del análisis arqueozoológico, tales como: preservación, recuperación e identificación de restos, así como de la fiabilidad de la información etnozoológica.

Para establecer este análisis comparativo diacrónico sobre los patrones de uso de la fauna por parte de las sociedades, se han establecido categorías como la de pérdida y la de incorporación de fauna. Esto permite sugerir la presencia de posibles cambios de actitud cultural hacia ciertos organismos, los cuales implican abandono o incorporación de organismos como parte de los recursos aprovechados por las comunidades.

En la muestra analizada, se observa el caso particular de los roedores y anfibios aprovechados como parte de la dieta: los datos etnozoológicos nos muestran que esta no es una práctica fuera de lo común y, por tanto, que su interpretación como parte del registro arqueozoológico debe ser reevaluada, considerándolos también como parte de los recursos consumidos.

Cabe destacar que hay una tercera categoría en el análisis diacrónico: la persistencia en el aprovechamiento de esos recursos faunísticos. Esta permite establecer una hipótesis inicial sobre el núcleo básico de aprovechamiento animal y las variantes que puede adquirir debido a las condiciones regionales.

En tal sentido, se considera que este tipo de estudios comparativos, circunscritos a un escenario geográfico determinado, nos ofrecen la oportunidad de explorar hipótesis de trabajo que pueden ser promisorias en la evaluación del impacto histórico de las sociedades sobre el ambiente. 
Agradecimientos: Al Laboratorio de Arqueozoología del Instituto Nacional de Antropología e Historia, donde originalmente se realizó este trabajo, por facilitarnos el acceso a sus archivos y colecciones. Al Centro INAH Morelos por las diversas facilidades otorgadas para dar conclusión a este trabajo.

\section{Referencias bibliográficas}

Acosta, Joseph

1962 Historia natural y moral de las Indias. México: Fondo de Cultura Económica.

Agrigner, Pierre

1975 Mound 1A, Chiapa de Corzo, Chiapas, Mexico: A Late Preclassic Architectural Complex. New World Archaeological Foundation Paper 39. Provo: Brigham Young University.

Álvarez, Ticul

1976 «Restos óseos de la primer excavación de Santa Marta, Chiapas». Informe de trabajo, Archivo del Laboratorio de Arqueozoología, Instituto Nacional de Antropología e Historia, México.

1979 «Estudio de los restos óseos procedentes de las dos temporadas en Corral de Piedra, Chiapas». Informe de trabajo, Archivo del Laboratorio de Arqueozoología, Instituto Nacional de Antropología e Historia, México.

1980 «Fogótico (Pozo 3). San Cristóbal de las Casa, Chiapas». Informe de trabajo, Archivo del Laboratorio de Arqueozoología, Instituto Nacional de Antropología e Historia, México.

1982 «Análisis del material zoológico de las excavaciones de Tonina, Chiapas», en Tonina. Une cité maya du Chiapas, Pierre Becquelin y Claude Baudez, coords., pp. 1127-1142. México: Mission Archéologique et Ethnologique Française au Mexique.

Álvarez, Ticul, Aurelio Ocaña y Norma Valentín

1980 «Identificación de los restos óseos procedentes de las excavaciones de Tonina, Chiapas». Informe de trabajo, Archivo del Laboratorio de Arqueozoología, Instituto Nacional de Antropología e Historia, México.

1990 «Identificación de los restos óseos provenientes de las excavaciones de Tonina, Chiapas», en Tonina. Une cité maya du Chiapas Vol. II, Pierre Becquelin y Claude Baudez, coords., pp. 1832-1846. México: Mission Archéologique et Ethnologique Française au Mexique.

BARRAGÁn, Felipe

2001 Roedores: un recurso alimentario en el municipio de Oxchuc, Chiapas. Tesis de Licenciatura. México: Instituto Tecnológico de Hidalgo.

ChÁvez A., Ernesto

1969 «Artifactual and Non-artifactual Material of the Phyla Mollusca, Arthropoda, and Chordata from Chiapa de Corzo, Chiapas», en The Artifacts of Chiapa de Corzo, Chiapas, Mexico, Thomas A. Lee, ed., pp. 219-220. Papers of the New World Archaeological Foundation 26. Provo: Brigham Young University.

Clarke K., Robert y Richard M. WARWICK

1998 «A Taxonomic Distinctness Index and its Statistical Properties». Journal of Applied Ecology 35: 523-531. 
Corona-M., Eduardo

2005 «Archaeozoology and the Role of Birds in the Traditional Medicine of Pre-Hispanic Mexico». Documenta Archaeobiologiae 3: 293-300.

2008 «Zoogeographical affinities and the use of vertebrates in Xochicalco (Morelos, Mexico)». Quaternary International 180: 145-151.

Corona-M., Eduardo y Joaquín Arroyo CABrales

2003 «Las relaciones hombre-fauna, una zona interdisciplinaria de estudio», en Relaciones hombre-fauna, una zona interdisciplinaria de estudio, Eduardo Corona-M. y Joaquín Arroyo-Cabrales, eds., pp. 17-28, México: Plaza y Valdéz - CONACULTA - INAH.

2007 «Human-Faunal Relationships: A Look from Paleoecology to Taphonomy», en Human and Faunal Relationships Reviewed: an Archaeozoological Approach, Eduardo Corona-M. y Joaquín Arroyo-Cabrales, eds., pp. 1-3. BAR International Series 1627. Oxford: Oxbow Books.

EMERY, Kitty F.

2004a «Historical Perspectives on Current Research Directions», en Maya Zooarchaeology: New Directions in Method and Theory, K. F. Emery, ed., pp. 1-14. Los Ángeles: Cotsen Institute of Archaeology at UCLA.

2004b «In Search of Assemblage Comparability. Methods in Maya Zooarchaeology», en Maya Zooarchaeology: New Directions in Method and Theory, K. F. Emery, ed., pp. 15-34. Los Ángeles: Cotsen Institute of Archaeology at UCLA.

ENRíQueZ, Patricia

2005 Uso medicinal de la fauna silvestre en los Altos de Chiapas. Tesis de Maestría. México: Colegio de la Frontera Sur.

EnRíluez, Patricia, Ramón Mariaca, Óscar Retana y Eduardo NARAnjo

2006 «Uso medicinal de la fauna silvestre en los Altos de Chiapas». Interciencia 31: 491-499.

FLANNERY, Kent V.

1969 «An Analysis of Animal Bones from Chiapa de Corzo, Chiapas», en The Artifacts of Chiapa de Corzo, Chiapas, Mexico, Thomas A. Lee, ed., pp. 209-218. Papers of the New World Archaeological Foundation 26. Provo: Brigham Young University.

FLORES-VILLELA, Óscar

1993 «Herpetofauna mexicana. Lista anotada de las especies de anfibios y reptiles de México, cambios taxonómicos recientes y nuevas especies». Carnegie Museum of Natural History, Special Publication 7: 1-73.

GARCía BÁrCEnA, Joaquín

1982 El precerámico de Aguacatenango, Chiapas, México. México: Instituto Nacional de Antropología e Historia.

Green Dee F. y Gareth W. Lowe

1967 Altamira and Padre Piedra, Early Preclassic Sites in Chiapas Mexico. Papers of the New World Archaeological Foundation 20. Provo: Brigham Young University.

Guerra, Michelle

2001 Cacería de subsistencia en dos localidades de la Selva Lacandona, Chiapas, México. Tesis de Licenciatura. México: Universidad Nacional Autónoma de México. 
HAMMER, Øyvind

2009 Paleontological Statistics Ver. 1.98. Reference Manual. Oslo: Natural History Museum, Oslo University. Documento electrónico, <http://folk.uio.no/ohammer/ past>, con acceso el 29/1/2010.

HARRIS David, R.

2006 «The Interplay of Ethnographic and Archaeological Knowledge in the Study of Past Human Subsistence», en Ethnobiology and the Science of Human Kind, Roy Ellen, ed., pp. 63-78. Londres: Blackwell.

Howell, Steve N. G. y Sophie WebB

1995 A Guide to the Birds of Mexico and Central America. Oxford: Oxford University Press.

Marmolejo, Miguel A.

2000 Fauna alimentaría de la península de Yucatán. México: Instituto Nacional Indigenista.

MARrinAn, Rochelle A.

1986 «Appendix: Faunal Analysis», en Early and Middle Preclassic Sub-Mound Refuse Deposit at Vistahermosa, Chiapas, Raymond C. Treat, ed., pp. 26-37. Notes on the New World Archaeological Foundation, Vol. 2, Provo: Brigham Young University.

MARION, Marie Odile

1994 Identidad y ritualidad entre los mayas. México: Instituto Nacional Indigenista.

Martínez Muriel, Alejandro

1989 «Basureros del Formativo Tardío en Don Martín, Chiapas». Arqueología 2a época, 1: $61-70$.

OCAÑA, Aurelio

1997 «Estudio de los mamíferos del Templo Olvidado, Palenque, Chiapas», en Homenaje al profesor Ticul Álvarez, Joaquín Arroyo Cabrales y Óscar J. Polaco eds., pp. 239-252. México: Instituto Nacional de Antropología e Historia.

Ocaña, Aurelio y Óscar J. POLACO

1982 «Informe de los restos óseos procedentes de Tonina, Chiapas». Informe de trabajo, Archivo del Laboratorio de Arqueozoología, Instituto Nacional de Antropología e Historia, México.

1990 «Informe de restos óseos procedentes de Tonina, Chiapas», en Tonina. Une cité maya du Chiapas Vol. II, Pierre Becquelin y Claude Baudez, coords., vol. 2, pp. 1851-1852. México: Mission Archéologique et Ethnologique Française au Mexique.

Polaco, Óscar J.

1989 «Los vertebrados de Laguna de Miramar, Chiapas». Informe de trabajo, Archivo del Laboratorio de Arqueozoología, Instituto Nacional de Antropología e Historia, México.

ReID, Fiona A.

1997 A Field Guide to the Mammals of Central America and Southeast of Mexico. Oxford: Oxford University Press.

SÁnchez Alvarez, Miguel

2000 Los tzotziles-tzeltales y su relación con la fauna silvestre. México: Consejo para la Cultura y las Artes de Chiapas. 
Soto ToRal, Heriberto

1998 Estudio arqueozoológico en la ciudad prehispánica maya de Yaxchilan, Chiapas. Tesis de Licenciatura, México: Instituto Politécnico Nacional.

Soto Hall, Máximo

1941 Cultura maya. Carácter y creaciones de esta gran civilización precolombina. México: Ediciones Atlántida.

VoorhiEs, Barbara

1976 The Chantuto People: An Archaic Period Society of the Chiapas Littoral, Mexico. Papers of the New World Archaeology Foundation, 41. Provo: Brigham Young University. 